\title{
Sequence and Simple-Sequence Repeat Analyses of the Fungal Pathogen Seiridium cardinale Indicate California Is the Most Likely Source of the Cypress Canker Epidemic for the Mediterranean Region
}

\author{
G. Della Rocca, C. A. Eyre, R. Danti, and M. Garbelotto
}

First author: Fondazione Edmund Mach-Istituto Agrario di san Michele all'Adige, via E. Mach, 38010 San Michele all'Adige (TN), Italy; first and third authors: Institute for Plant Protection-CNR, Via Madonna del Piano 10, I-50019, Sesto Fiorentino (FI), Italy; and second and fourth authors: Department of ESPM, 137 Mulford Hall, University of California, Berkeley 94720.

Accepted for publication 3 August 2011.

\section{ABSTRACT}

Della Rocca, G., Eyre, C. A., Danti, R., and Garbelotto, M. 2011. Sequence and simple-sequence repeat analyses of the fungal pathogen Seiridium cardinale indicate California is the most likely source of the cypress canker epidemic for the Mediterranean region. Phytopathology 101:1408-1417.

Seiridium cardinale is the pathogenic fungus of unknown origin responsible for a world pandemic known as cypress canker affecting several species of Cupressaceae in both the Northern and Southern Hemisphere. In this study, a comparative genetic analysis of worldwide populations was performed using sequence analysis of a portion of the $\beta$ tubulin locus and seven polymorphic simple-sequence repeat (SSR) loci on 96 isolates. Sequence analysis identified two distinct $\beta$-tubulin alleles, both present in California. Only one of the two alleles was detected in the Mediterranean basin, while two isolates from the Southern Hemisphere were characterized by the presence of the allele absent from the Mediterranean. SSRs identified a total of 46 multilocus genotypes (MGs): genotypic diversity was always higher in the California population, and calculations of the index of association $\left(\mathrm{I}_{\mathrm{A}}\right)$ determined the presence of linkage disequilibrium associated with the absence of sexual reproduction only in the Mediterranean population but not in California. In 50 instances, the same MG was found at great geographic distances, implying a role played by humans in spreading the disease. Network analysis performed on SSR data identified three clusters of MGs: California, Morocco, and the rest of the Mediterranean. Both the Morocco and the Mediterranean clusters were linked to the California cluster. Coalescent analysis identified insignificant migration between California and Italy, as expected in the presence of a single introduction event, and very high migration from Italy into Greece, as expected of an outbreak still in exponential growth phase and starting from an Italian source.

Additional keywords: coalescent analysis, Cupressus, genetic diversity, microsatellite, network analysis.
Seiridium cardinale (W. W. Wagener) B. Sutton \& I. A. S. Gibson is the pathogenic fungus responsible for a world pandemic known as cypress canker. Although two other Seiridium spp. (namely, S. unicorne and $S$. cupressi) can also cause a similar disease, their overall abundance and virulence are clearly lower when compared with those of $S$. cardinale (30). Disease symptoms include lethal bark canker on many members of the family Cupressaceae, including Cupressus, Thuja, Juniperus, Calocedrus, and Chamaecyparis spp. and the intergeneric hybrid $\times$ Cupressocyparis leylandii (Chamaecyparis nootkatensis $q \times$ Cupressus macrocarpa $\widehat{\jmath})(26,34,61,71,72)$. Cankers may expand relatively slowly on the main stem but can be numerous and relatively rapid-growing on branches, resulting in visible dieback of different portions of the tree canopy that eventually lead to tree mortality. Where high density of susceptible species exist and in the presence of warm and humid conditions known to favor spread of the disease, infection and mortality levels can be $\leq 95 \%$, with obvious ecological and economic consequences $(50,74)$. Cypress canker has had a major economic impact during its worldwide spread of the last 50 years; in fact, cypress trees are not only important components of natural ecosystems but are also highly valued ornamental species and represent some of the most common trees employed to create windbreaks for agricultural pur-

Corresponding author: M. Garbelotto; E-mail address: matteog @ berkeley.edu

doi:10.1094/PHYTO-05-11-0144

(c) 2011 The American Phytopathological Society poses. In the Mediterranean region, cypress trees also have a great cultural relevance, making the impact of this disease particularly worrisome $(30,49)$.

The first epidemic of cypress canker caused by $S$. cardinale was reported in 1928 in California and resulted in the quick demise of many plantations of $C$. macrocarpa in the interior areas of the state, where this tree species does not occur naturally $(74,75)$. Since this initial report, the disease has been described in New Zealand (12), Europe $(2,6,15,33,66,69)$, Australia (68), South America $(4,46,61)$, South Africa (76), and North Africa $(18,25)$ as a true pandemic $(30)$. The sudden recent appearances of the disease and the extremely high levels of mortality experienced in many areas around the globe suggest that the disease and its causal agent are not cosmopolitan by ancient history but, rather, have been introduced into most locations, presumably through human activities. Because cypress trees are traded widely as ornamental plants, this remains the most plausible means of transport across continents. Alternatively, the movement of infected cypress wood may also play a role in the long-distance movement of the pathogen. However, even though the fungus can survive for a long period in the wood of cypress without loss of pathogenicity, sporulation on dead wood or timber in use is reported to be unlikely $(47,51)$; hence, the pathogen introduced in wood may not be able to spread from the initial introduction point.

Various hypotheses have been formulated on the possible origin of the disease but none of them have been substantiated by direct or even corroborating evidence. The first hypothesis is that $S$. cardinale might exist as a weak pathogen on some as-yet-un- 
identified hosts in the western United States and that plantings of C. macrocarpa away from its natural coastal range may have facilitated a host jump. The second hypothesis suggests that the pathogen may have arisen as a variant (mutation or hybridization) from a normally saprophytic indigenous fungus. A third possibility implies a source other than California and a dispersal pattern to other regions of the world that may or may not go through California (75). For instance, the fungus could be native to East or South Asia where all indigenous Cupressus spp. are characterized by a high degree of resistance to $S$. cardinale (53, 55). The plausibility of this hypothesis is supported, in part, by the early distribution of the disease in Pacific-rim states such as California (75), New Zealand (12), and Chile (in 46).

Identifying the area of origin of the pathogen and its routes of spread is of paramount importance. Studying native populations of the pathogen in the ecosystem in which they have coevolved with their hosts may help better understand the biology and epidemiology of the pathogen, and may further allow the identification of the various evolutionary strategies that have led to resistance in plants. More urgently, understanding the source of the pathogen and its mode of spread may help prevent both the introduction of the pathogen in new areas and the introduction of substantially different pathogen genotypes into areas where the pathogen has already arrived. This last aspect is particularly relevant because the most valuable form of control lies in the resistant cypress lines that have already been developed $(3,19$, $50,58,59)$, a strategy and a 30 -year-long effort that could be nullified by the introduction of substantially different genotypes of the pathogen.

A first necessary approach to infer the source of a pathogen is to perform a comparative analysis of the genetic diversity of populations from different areas and determine the relationships among these populations. The hypothesis is that source populations should be more diverse while derived populations should be less diverse and nested within source populations. Similar analyses have been successfully done for many exotic forest diseases, including chestnut blight, sudden oak death, white pine blister rust, canker stain of plane, and North American Heterobasidion root rot in Italy $(22,23,28,32,35,39,42,48)$. Additionally, genetic analyses allow us to infer whether sexual reproduction may be effectively ongoing in a population. This aspect is particularly relevant not only for its epidemiological and co-evolutionary implications but also because sexual reproduction in most fungal pathogens requires the presence of at least two mating types. Historically, many introduced forest diseases are locally characterized by the presence of a single mating type, whereas both mating types are normally present in the area of origin (unless, of course, the organism in question has lost its ability to reproduce sexually) or when both mating types of a pathogen are introduced. $S$. cardinale is considered to be an anamorphic fungus reproducing agamically through conidia made of haploid cells. The genus Leptosphaeria, the perfect stage of $S$. cardinale, was reported only once on dying and dead cypress trees over a large area of north central California but it was only partially described (33), and it has been deemed too incomplete to be fully recognized.

Very limited information is available on variability of $S$. cardinale within and between regions. Published reports either focus on $S$. cardinale genotypes from a single region or focus on the evolutionary relationships between $S$. cardinale and its sister species. If we exclude the very preliminary tests described in the first report on this pathogen (75), most analyses of variation within $S$. cardinale have been performed with regard to virulence and exclusively on a few European isolates. Results have indicated a substantial uniformity among isolates of the fungus, in agreement with the expectations for an introduced exotic species $(55,57,77)$. Only a few studies have used molecular techniques to look at genetic diversity within Seiridium isolates. Most studies have focused on comparisons among rather than within species $(5,36,45,60,71,73)$. With the exception of Viljoen et al. (73), who suggested the existence of a single, morphologically, variable species, these studies all supported the distinction of $S$. cardinale, $S$. cupressi, and $S$. unicorne as separate species; however, all failed to detect variability among $S$. cardinale isolates. Randomly amplified polymorphic DNAs indicated a high level of homogeneity among $77 \mathrm{~S}$. cardinale isolates from northern Italy and 1 isolate from Greece (53) but the lack of representation of a region outside of Europe in the sample precluded any conclusions on the origin of the pathogen or on the source of the European population.

In this study, we analyze $\beta$-tubulin sequences (36) and seven polymorphic simple-sequence repeats (SSRs) (20) on a total of 96 isolates of $S$. cardinale from California, several Mediterranean countries, Chile, and New Zealand. We use sequence analysis to confirm identity of the isolates employed in the study and to search for a worldwide pattern in distribution of haplotypes. SSR data instead are used to (i) determine presence of linkage disequilibrium (LD) in the data set to verify the potential for presence of cryptic sex, (ii) calculate indices of genotypic and genetic diversity inside and outside California, and, (iii) perform a coalescent analysis and a minimum spanning network (MSN) to highlight the relationship between California and Mediterranean isolates, as well as intraregional relationships. Results clearly indicate that California is the most likely source of two distinct infestations in the Mediterranean and a possible candidate for the area of origin of this pathogen. Additionally, distribution of genotypes is consistent with long-distance human-mediated movement of the pathogen. Implications for the future of the susceptible hosts and for trade regulations will be discussed.

\section{MATERIALS AND METHODS}

Sampling. A total of 96 Seiridium cardinale isolates was obtained in 2007 to 2008 through a sampling effort of symptomatic Cupressaceae spp. from seven Mediterranean countries and eight Californian counties (Table 1). Additionally, $18 \beta$ tubulin sequences ( 8 of $S$. cardinale, 3 of $S$. unicorne, and 7 of $S$. cupressi) from GenBank (10) were also included in the neighborjoining (NJ) analysis. GenBank accession numbers of sequences not generated in this study are provided in the taxon labels of Figure 1.

Fungal isolation. Small bark fragments $(\approx 3$ by $3 \mathrm{~mm})$ cut from the margin of cankered tissues were placed on potato dextrose agar (PDA) (potato dextrose broth [PDB] at $20 \mathrm{~g} / \mathrm{liter}+$ agar at $20 \mathrm{~g} /$ liter) in petri dishes and maintained at $25^{\circ} \mathrm{C}$ in the dark. White to gray or olive, cottony colonies with a salmon-orange reverse side, obtained from plated fragments, were separately transferred on $1 \%$ malt extract agar (malt extract at $10 \mathrm{~g} /$ liter + agar at $20 \mathrm{~g} /$ liter) supplemented with autoclaved cypress seed. The plates were then incubated at $18^{\circ} \mathrm{C}$ under mixed white and near-ultraviolet light (400 to $200 \mathrm{~nm}$ ), set to provide 12-h lightand-dark cycles to induce sporulation of the fungus. After 3 to 4 weeks, monoconidial cultures were obtained from acervuli (i.e., asexual sporulating structures producing mitospores known as conidia) of the fungus developed on seed $(16,44,68,75)$. Seed bearing acervuli of each isolate were collected and stored in hermetic plastic tubes at $-20^{\circ} \mathrm{C}$.

DNA extraction and polymerase chain reaction amplification. For DNA extraction, the cultures were grown on cellophane discs placed on PDA (PDB at $20 \mathrm{~g} / \mathrm{liter}+$ agar at $20 \mathrm{~g} / \mathrm{liter}$ ) in petri dishes for 15 to 20 days at $25^{\circ} \mathrm{C}$. The colonies were then lifted from cellophane and freeze dried for $24 \mathrm{~h}$. Genomic DNA was extracted using the DNeasy Plant Mini Kit (Qiagen, Hilden, Germany) in agreement with the indications of the producer.

A portion of the $\beta$-tubulin locus was amplified for a subset of 21 California and 5 Mediterranean isolates using primers Bt1a 
and Bt $1 \mathrm{~b}$ and conditions described by Barnes et al. (5). The amplicons were sequenced on an Applied Biosystems 3130xl Genetic Analyzer (Life Technologies, Carlsbad, CA). Seven polymorphic nuclear microsatellites developed and characterized for $S$. cardinale (20) were used in this study. Polymerase chain reaction (PCR) mixtures and cycling conditions were the same as those described by Della Rocca et al. (20).

Analyses. Sequences were manually edited and aligned using Sequencher 4.2 (Gene Codes Corporation, Ann Arbor, MI) before being analyzed using Geneious Pro 5.1.6. (21) (Biomatters Ltd., Auckland, New Zealand). An NJ tree was generated for the selected isolates, including sequences of isolates of $S$. cardinale from Chile and New Zealand deposited in GenBank (AF320504.1 and AF320500.1). Isolates of S. cupressi and S. unicorne were used as outgroups. The Tamura-Nei genetic distance model was used and bootstrap support was calculated with 1,000 replicates. Additionally, sequences were exported as FastA-formatted files, which were converted to a "relaxed" Phylip format (i.e., allowing taxon labels to occupy $\leq 256$ characters) using a Perl script available on the webpage of A. Stamatakis (http://www.kramer. in.tum.de/exelixis/software.html). Maximum likelihood (ML) phylogenetic analyses were performed using RAxML-HPC2 v7.2.8 (64) implemented on the CIPRES Portal v2.2 at the San Diego Supercomputer Center (43). Analyses included 200 inferences on the original alignment and 1,000 bootstrap replicates, using the GTR+GAMMA model for both bootstrapping and final ML optimization and using default parameter settings. Trees were visualized with FigTree v1.3.1 (http://tree.bio.ed.ac.uk/ software/figtree). All sequences generated in the course of this study were deposited in GenBank (Table 1).

Size of amplicons obtained with primers flanking SSRs was transformed to number of repeats and used for all further analyses. The index of association $\mathrm{I}_{\mathrm{A}}$ was calculated using the program MULTILOCUS v1.3 (1) independently for isolates from California and from the Mediterranean, excluding Moroccan genotypes which were characterized by significantly different allelic sizes than all other genotypes from the same region (see Results). $\mathrm{I}_{\mathrm{A}}$ is a measure of $\mathrm{LD}$ - the number of loci at which pairs of individuals differ; this pairwise "distance" is calculated and its variance compared with that if there was no LD.

TABLE 1. Summary of Seiridium cardinale sampling locations

\begin{tabular}{|c|c|c|c|c|c|c|c|}
\hline Isolate & Host & Provenance & Country & Latitude N & Longitude & $\begin{array}{l}\text { Multilocus } \\
\text { genotype }\end{array}$ & $\begin{array}{c}\text { GenBank } \beta \text {-tubulin } \\
\text { accession }\end{array}$ \\
\hline 430 & Cupressus sempervirens & Algiers & Algeria & $36^{\circ} 45^{\prime} 21^{\prime \prime}$ & $3^{\circ} 02^{\prime} 45^{\prime \prime}$ & MG36 & $\ldots$ \\
\hline 431 & C. sempervirens & Algiers & Algeria & $36^{\circ} 45^{\prime} 21^{\prime \prime}$ & $3^{\circ} 02^{\prime} 45^{\prime \prime}$ & MG35 & $\ldots$ \\
\hline 92 & C. sempervirens & Corte & France & $42^{\circ} 18^{\prime} 20^{\prime \prime}$ & $9^{\circ} 09^{\prime} 03^{\prime \prime}$ & MG33 & $\ldots$ \\
\hline 57 & C. sempervirens & Bastia & France & $42^{\circ} 42^{\prime} 03^{\prime \prime}$ & $9^{\circ} 26^{\prime} 56^{\prime \prime}$ & MG30 & $\ldots$ \\
\hline 176 & C. sempervirens & Frejus & France & $43^{\circ} 25^{\prime} 27^{\prime \prime}$ & $6^{\circ} 43^{\prime} 26^{\prime \prime}$ & MG30 & $\ldots$ \\
\hline 181 & C. sempervirens & Frejus & France & $43^{\circ} 25^{\prime} 27^{\prime \prime}$ & $6^{\circ} 43^{\prime} 26^{\prime \prime}$ & MG27 & $\ldots$ \\
\hline 251 & C. sempervirens & Amamine & France & $43^{\circ} 57^{\prime} 57^{\prime \prime}$ & $4^{\circ} 12^{\prime} 49^{\prime \prime}$ & MG30 & HQ678148 \\
\hline 150 & C. sempervirens & Omalos & Greece & $35^{\circ} 20^{\prime} 38^{\prime \prime}$ & $23^{\circ} 54^{\prime} 17^{\prime \prime}$ & MG39 & $\ldots$ \\
\hline $150 \mathrm{~b}$ & C. sempervirens & Omalos & Greece & $35^{\circ} 20^{\prime} 38^{\prime \prime}$ & $23^{\circ} 54^{\prime} 17^{\prime \prime}$ & MG43 & $\ldots$ \\
\hline 335 & C. sempervirens & Nipos & Greece & $35^{\circ} 22^{\prime} 45^{\prime \prime}$ & $24^{\circ} 10^{\prime} 10^{\prime \prime}$ & MG42 & $\ldots$ \\
\hline 336 & C. sempervirens & Nipos & Greece & $35^{\circ} 22^{\prime} 45^{\prime \prime}$ & $24^{\circ} 10^{\prime} 10^{\prime \prime}$ & MG42 & $\ldots$ \\
\hline 337 & C. sempervirens & Nipos & Greece & $35^{\circ} 22^{\prime} 45^{\prime \prime}$ & $24^{\circ} 10^{\prime} 10^{\prime \prime}$ & MG42 & $\ldots$ \\
\hline 344 & C. sempervirens & Agrokepio & Greece & $35^{\circ} 18^{\prime} 55^{\prime \prime}$ & $24^{\circ} 12^{\prime} 42^{\prime \prime}$ & MG30 & $\ldots$ \\
\hline 345 & C. sempervirens & Agrokepio & Greece & $35^{\circ} 18^{\prime} 55^{\prime \prime}$ & $24^{\circ} 12^{\prime} 42^{\prime \prime}$ & MG40 & $\ldots$ \\
\hline 375 & C. sempervirens & Fres & Greece & $35^{\circ} 22^{\prime} 54^{\prime \prime}$ & $24^{\circ} 08^{\prime} 42^{\prime \prime}$ & MG30 & $\ldots$ \\
\hline 368 & C. sempervirens & Karistos & Greece & $38^{\circ} 00^{\prime} 57^{\prime \prime}$ & $24^{\circ} 24^{\prime} 41^{\prime \prime}$ & MG40 & $\ldots$ \\
\hline 366 & C. sempervirens & Karistos & Greece & $38^{\circ} 00^{\prime} 57^{\prime \prime}$ & $24^{\circ} 24^{\prime} 41^{\prime \prime}$ & MG42 & $\ldots$ \\
\hline 404 & C. sempervirens & M. Athos & Greece & $40^{\circ} 26^{\prime} 00^{\prime \prime}$ & $23^{\circ} 50^{\prime} 33^{\prime \prime}$ & MG31 & $\ldots$ \\
\hline 191 & C. sempervirens & Kaliani & Greece & $37^{\circ} 53^{\prime} 18^{\prime \prime}$ & $22^{\circ} 29^{\prime} 50^{\prime \prime}$ & MG44 & $\ldots$ \\
\hline 359 & C. sempervirens & Kaliani & Greece & $37^{\circ} 53^{\prime} 18^{\prime \prime}$ & $22^{\circ} 29^{\prime} 50^{\prime \prime}$ & MG30 & $\ldots$ \\
\hline 192 & Juniperus phoenicea & Lefkas & Greece & $38^{\circ} 49^{\prime} 49^{\prime \prime}$ & $20^{\circ} 42^{\prime} 25^{\prime \prime}$ & MG34 & $\ldots$ \\
\hline 194 & C. sempervirens & Spanochori & Greece & $38^{\circ} 47^{\prime} 00^{\prime \prime}$ & $20^{\circ} 40^{\prime} 18^{\prime \prime}$ & MG34 & $\ldots$ \\
\hline 196 & C. sempervirens & Hortata & Greece & $38^{\circ} 43^{\prime} 01^{\prime \prime}$ & $20^{\circ} 36^{\prime} 09^{\prime \prime}$ & MG30 & $\ldots$ \\
\hline 314 & C. sempervirens & Megalopoli & Greece & $37^{\circ} 24^{\prime} 03^{\prime \prime}$ & $22^{\circ} 08^{\prime} 28^{\prime \prime}$ & MG37 & $\ldots$ \\
\hline 322 & C. sempervirens & Kalimani & Greece & $38^{\circ} 06^{\prime} 55^{\prime \prime}$ & $22^{\circ} 57^{\prime} 08^{\prime \prime}$ & MG31 & $\ldots$ \\
\hline 319 & C. sempervirens & Kalimani & Greece & $38^{\circ} 06^{\prime} 55^{\prime \prime}$ & $22^{\circ} 57^{\prime} 08^{\prime \prime}$ & MG30 & HQ678149 \\
\hline 323 & C. sempervirens & Kritharion & Greece & $38^{\circ} 02^{\prime} 44^{\prime \prime}$ & $21^{\circ} 43^{\prime} 27^{\prime \prime}$ & MG30 & $\ldots$ \\
\hline 325 & C. sempervirens & Kritharion & Greece & $38^{\circ} 02^{\prime} 44^{\prime \prime}$ & $21^{\circ} 43^{\prime} 27^{\prime \prime}$ & MG30 & $\ldots$ \\
\hline 334 & C. sempervirens & Alepochori & Greece & $37^{\circ} 59^{\prime} 01^{\prime \prime}$ & $21^{\circ} 47^{\prime} 54^{\prime \prime}$ & MG30 & $\ldots$ \\
\hline 333 & C. sempervirens & Alepochori & Greece & $37^{\circ} 59^{\prime} 01^{\prime \prime}$ & $21^{\circ} 47^{\prime} 54^{\prime \prime}$ & MG36 & $\ldots$ \\
\hline 328 & C. sempervirens & Nea Makri & Greece & $38^{\circ} 04^{\prime} 54^{\prime \prime}$ & $23^{\circ} 58^{\prime} 57^{\prime \prime}$ & MG30 & $\ldots$ \\
\hline 331 & C. sempervirens & Nea Makri & Greece & $38^{\circ} 04^{\prime} 54^{\prime \prime}$ & $23^{\circ} 58^{\prime} 57^{\prime \prime}$ & MG29 & $\ldots$ \\
\hline 403 & C. sempervirens & Nea Makri & Greece & $38^{\circ} 04^{\prime} 54^{\prime \prime}$ & $23^{\circ} 58^{\prime} 57^{\prime \prime}$ & MG31 & $\ldots$ \\
\hline 70 & C. sempervirens & Montecavolo & Italy & $44^{\circ} 37^{\prime} 54^{\prime \prime}$ & $10^{\circ} 32^{\prime} 11^{\prime \prime}$ & MG24 & $\ldots$ \\
\hline 118 & C. sempervirens & Bellaria & Italy & $44^{\circ} 08^{\prime} 22^{\prime \prime}$ & $12^{\circ} 28^{\prime} 24^{\prime \prime}$ & MG30 & $\ldots$ \\
\hline 253 & $\times$ Cupressocyparis leylandii & Imperia & Italy & $43^{\circ} 53^{\prime} 57^{\prime \prime}$ & $8^{\circ} 02^{\prime} 17^{\prime \prime}$ & MG30 & $\ldots$ \\
\hline 249 & Cupressus sempervirens & Platamona & Italy & $40^{\circ} 48^{\prime} 07^{\prime \prime}$ & $8^{\circ} 28^{\prime} 40^{\prime \prime}$ & MG30 & $\ldots$ \\
\hline 56 & C. sempervirens & Sassari & Italy & $40^{\circ} 42^{\prime} 49^{\prime \prime}$ & $8^{\circ} 31^{\prime} 41^{\prime \prime}$ & MG30 & $\ldots$ \\
\hline 53 & C. sempervirens & Troina & Italy & $37^{\circ} 47^{\prime} 42^{\prime \prime}$ & $14^{\circ} 36^{\prime} 35^{\prime \prime}$ & MG23 & $\ldots$ \\
\hline 105 & C. sempervirens & Comiso & Italy & $36^{\circ} 56^{\prime} 44^{\prime \prime}$ & $14^{\circ} 36^{\prime} 14^{\prime \prime}$ & MG22 & $\ldots$ \\
\hline $105 b$ & C. sempervirens & Comiso & Italy & $36^{\circ} 56^{\prime} 44^{\prime \prime}$ & $14^{\circ} 36^{\prime} 14^{\prime \prime}$ & MG22 & $\ldots$ \\
\hline 64 & C. sempervirens & Guarniente & Italy & $43^{\circ} 32^{\prime} 26^{\prime \prime}$ & $11^{\circ} 53^{\prime} 20^{\prime \prime}$ & MG30 & $\ldots$ \\
\hline 115 & C. sempervirens & Gargiano & Italy & $43^{\circ} 39^{\prime} 12^{\prime \prime}$ & $11^{\circ} 51^{\prime} 02^{\prime \prime}$ & MG27 & $\ldots$ \\
\hline 168 & C. sempervirens & S. Luce & Italy & $43^{\circ} 28^{\prime} 17^{\prime \prime}$ & $10^{\circ} 33^{\prime} 49^{\prime \prime}$ & MG30 & $\ldots$ \\
\hline 165 & C. sempervirens & Fosdinovo & Italy & $44^{\circ} 07^{\prime} 59^{\prime \prime}$ & $10^{\circ} 01^{\prime} 03^{\prime \prime}$ & MG30 & $\ldots$ \\
\hline $165 b$ & C. sempervirens & Fosdinovo & Italy & $44^{\circ} 07^{\prime} 59^{\prime \prime}$ & $10^{\circ} 01^{\prime} 03^{\prime \prime}$ & MG30 & $\ldots$ \\
\hline 164 & C. sempervirens & Carrara & Italy & $44^{\circ} 04^{\prime} 54^{\prime \prime}$ & $10^{\circ} 06^{\prime} 26^{\prime \prime}$ & MG40 & HQ678147 \\
\hline 167 & C. sempervirens & Bolgheri & Italy & $43^{\circ} 13^{\prime} 54^{\prime \prime}$ & $10^{\circ} 36^{\prime} 01^{\prime \prime}$ & MG29 & $\ldots$ \\
\hline
\end{tabular}


The following indices of diversity were calculated as described by Mascheretti et al. (38): (i) clonal genotype diversity, $R=(G-$ $1) /(N-1)$, where $G$ is the number of multilocus genotypes (MGs) present in a sample and $N$ is the sample size; (ii) Stoddart and Taylor's Index, $G=1 / \Sigma p_{i}^{2},(63)$ where $p_{i}$ is the frequency of the $i$ th MG; and (iii) gene diversity (expected heterozygosity), estimated as

$$
H_{E}=1-\frac{1}{m} \sum_{l=1}^{m} \sum_{i=1}^{k} p_{i}^{2}
$$

where $p$ is the frequency of the $i$ th of $k$ alleles, averaged over each $l$ of $m$ loci. Calculations were performed on the same groupings of isolates described above for $\mathrm{I}_{\mathrm{A}}$ analysis.

To avoid the limitations of phylogenetic approaches that inevitably result in bifurcated relationships among groups of genotypes, we opted to use the data instead to determine a MSN for the entire dataset based on the reconstruction of all possible linkages among genotypes. MGs were first determined by identifying unique combinations of alleles, and a pairwise matrix of genetic distances among all MGs was then calculated using the robust method of Bruvo et al. (14) in the program GenoDive
$2.0 \mathrm{~b} 10$ (40) using the stepwise mutation model. The program MINSPNET (24) was used to create a MSN that depicts the relationships among all MGs, based on Bruvo's distances, and visualized using Graphviz 2.26.3 (27) (http://www.graphviz.org).

MIGRATE-N v3.1.6 (8) was used to perform coalescent analyses and determine extent and direction of migration among the following regions: California, Italy, and Greece. The choice of these three regions for the coalescent analysis was dictated by (i) a significant number of genotypes available for each of these regions and (ii) the sequence of reports of the disease clearly described first in California, then in Italy, and only later in Greece and other Mediterranean regions. A Bayesian inference method was used $(7,9)$ to estimate direction migration rates between these three populations. Microsatellite evolution was modeled using the Brownian motion model; genealogies were started with the automatic unweighted pair-group method with arithmetic meansbased start; and an assumption of a constant mutation rate for all loci, burn in of 10,000, and adaptive heating with four chains were used in the analysis. Migration, $M$, was estimated as the immigration rate $m$ divided by the mutation rate $\mu$. Prior distribution parameters for $\theta$ and $M$ were assumed to be uniform for

TABLE 1. (continued from preceding page)

\begin{tabular}{|c|c|c|c|c|c|c|c|}
\hline Isolate & Host & Provenance & Country & Latitude N & Longitude & $\begin{array}{l}\text { Multilocus } \\
\text { genotype }\end{array}$ & $\begin{array}{c}\text { GenBank } \beta \text {-tubulin } \\
\text { accession }\end{array}$ \\
\hline $167 b$ & C. sempervirens & Bolgheri & Italy & $43^{\circ} 13^{\prime} 54^{\prime \prime}$ & $10^{\circ} 36^{\prime} 01^{\prime \prime}$ & MG29 & $\ldots$ \\
\hline 44 & C. sempervirens & Bolgheri & Italy & $43^{\circ} 13^{\prime} 54^{\prime \prime}$ & $10^{\circ} 36^{\prime} 01^{\prime \prime}$ & MG30 & $\ldots$ \\
\hline 402 & C. sempervirens & Firenze & Italy & $43^{\circ} 45^{\prime} 13^{\prime \prime}$ & $11^{\circ} 19^{\prime} 21^{\prime \prime}$ & MG38 & $\ldots$ \\
\hline 104 & C. sempervirens & Serpiolle & Italy & $43^{\circ} 49^{\prime} 14^{\prime \prime}$ & $11^{\circ} 15^{\prime} 09^{\prime \prime}$ & MG30 & $\ldots$ \\
\hline 76 & $\times$ Cupressocyparis leylandii & Calenzano & Italy & $43^{\circ} 51^{\prime} 06^{\prime \prime}$ & $11^{\circ} 10^{\prime} 05^{\prime \prime}$ & MG45 & $\ldots$ \\
\hline 178 & Cupressus sempervirens & San Gimignano & Italy & $43^{\circ} 28^{\prime} 04^{\prime \prime}$ & $11^{\circ} 02^{\prime} 25^{\prime \prime}$ & MG30 & $\ldots$ \\
\hline 179 & C. sempervirens & Larderello & Italy & $43^{\circ} 14^{\prime} 04^{\prime \prime}$ & $10^{\circ} 53^{\prime} 26^{\prime \prime}$ & MG30 & $\ldots$ \\
\hline 61 & C. sempervirens & Fonterutoli & Italy & $43^{\circ} 26^{\prime} 12^{\prime \prime}$ & $11^{\circ} 18^{\prime} 29^{\prime \prime}$ & MG30 & $\ldots$ \\
\hline 114 & C. sempervirens & Poggio d'Acona & Italy & $43^{\circ} 38^{\prime} 04^{\prime \prime}$ & $11^{\circ} 52^{\prime} 31^{\prime \prime}$ & MG31 & $\ldots$ \\
\hline 103 & C. sempervirens & Roselle & Italy & $42^{\circ} 48^{\prime} 52^{\prime \prime}$ & $11^{\circ} 07^{\prime} 30^{\prime \prime}$ & MG30 & $\ldots$ \\
\hline 170 & C. sempervirens & Saturnia & Italy & $42^{\circ} 39^{\prime} 48^{\prime \prime}$ & $11^{\circ} 30^{\prime} 39^{\prime \prime}$ & MG26 & $\ldots$ \\
\hline 172 & C. sempervirens & Arcidosso & Italy & $42^{\circ} 52^{\prime} 21^{\prime \prime}$ & $11^{\circ} 32^{\prime} 23^{\prime \prime}$ & MG32 & $\ldots$ \\
\hline 157 & C. sempervirens & Mori & Italy & $45^{\circ} 51^{\prime} 09^{\prime \prime}$ & $10^{\circ} 58^{\prime} 34^{\prime \prime}$ & MG40 & HQ678146 \\
\hline 156 & C. sempervirens & Ala & Italy & $45^{\circ} 45^{\prime} 25^{\prime \prime}$ & $11^{\circ} 00^{\prime} 01^{\prime \prime}$ & MG36 & $\ldots$ \\
\hline 158 & C. sempervirens & Mezzocorona & Italy & $46^{\circ} 12^{\prime} 50^{\prime \prime}$ & $11^{\circ} 07^{\prime} 11^{\prime \prime}$ & MG30 & $\ldots$ \\
\hline 73 & C. sempervirens & Lago Trasimeno & Italy & $43^{\circ} 11^{\prime} 45^{\prime \prime}$ & $12^{\circ} 07^{\prime} 49^{\prime \prime}$ & MG30 & $\ldots$ \\
\hline 159 & C. sempervirens & Cannara & Italy & $43^{\circ} 00^{\prime} 49^{\prime \prime}$ & $12^{\circ} 37^{\prime} 05^{\prime \prime}$ & MG28 & $\ldots$ \\
\hline 63 & C. sempervirens & Cannara & Italy & $43^{\circ} 00^{\prime} 49^{\prime \prime}$ & $12^{\circ} 37^{\prime} 05^{\prime \prime}$ & MG25 & $\ldots$ \\
\hline 154 & C. sempervirens & Fès-Ifrane & Morocco & $33^{\circ} 42^{\prime} 57^{\prime \prime}$ & $5^{\circ} 00^{\prime} 45^{\prime \prime} \mathrm{O}$ & MG46 & HQ678145 \\
\hline $154 b$ & C. sempervirens & Fès-Ifrane & Morocco & $33^{\circ} 42^{\prime} 57^{\prime \prime}$ & $5^{\circ} 00^{\prime} 45^{\prime \prime} \mathrm{O}$ & MG46 & $\ldots$ \\
\hline $154 \mathrm{c}$ & C. sempervirens & Fès-Ifrane & Morocco & $33^{\circ} 42^{\prime} 57^{\prime \prime}$ & $5^{\circ} 00^{\prime} 45^{\prime \prime} \mathrm{O}$ & MG46 & $\ldots$ \\
\hline 513 & C. sempervirens & Conde dos Arcos & Portugal & $38^{\circ} 46^{\prime} 14^{\prime \prime}$ & $9^{\circ} 06^{\prime} 31^{\prime \prime} \mathrm{O}$ & MG7 & $\ldots$ \\
\hline 514 & C. sempervirens & Museu da Cidade & Portugal & $38^{\circ} 42^{\prime} 45^{\prime \prime}$ & $9^{\circ} 08^{\prime} 01^{\prime \prime} \mathrm{O}$ & MG41 & $\ldots$ \\
\hline 160 & C. sempervirens & S. Marino & S. Marino & $43^{\circ} 56^{\prime} 19^{\prime \prime}$ & $12^{\circ} 26^{\prime} 37^{\prime \prime}$ & MG30 & $\ldots$ \\
\hline 250 & C. sempervirens & Naquera & Spain & $39^{\circ} 39^{\prime} 31^{\prime \prime}$ & $0^{\circ} 25^{\prime} 32^{\prime \prime}$ & MG30 & $\ldots$ \\
\hline 475 & C. macnabiana & Berkeley & California & $37^{\circ} 52^{\prime} 27^{\prime \prime}$ & $122^{\circ} 14^{\prime} 18^{\prime \prime} \mathrm{O}$ & MG17 & HQ678150 \\
\hline 476 & C. macrocarpa & Berkeley Marina & California & $37^{\circ} 51^{\prime} 52^{\prime \prime}$ & $122^{\circ} 19^{\prime} 02^{\prime \prime} \mathrm{O}$ & MG4 & HQ678151 \\
\hline 477 & C. macrocarpa & Berkeley Marina & California & $37^{\circ} 51^{\prime} 52^{\prime \prime}$ & $122^{\circ} 19^{\prime} 02^{\prime \prime} \mathrm{O}$ & MG10 & $\ldots$ \\
\hline 485 & C. macrocarpa & Berkeley & California & $37^{\circ} 51^{\prime} 32^{\prime \prime}$ & $122^{\circ} 17^{\prime} 55^{\prime \prime} \mathrm{O}$ & MG11 & HQ678158 \\
\hline 483 & C. sempervirens & Lafayette & California & $37^{\circ} 53^{\prime} 42^{\prime \prime}$ & $122^{\circ} 07^{\prime} 18^{\prime \prime} \mathrm{O}$ & MG5 & HQ678156 \\
\hline 484 & $\times$ Cupressocyparis leylandii & Lafayette & California & $37^{\circ} 53^{\prime} 45^{\prime \prime}$ & $122^{\circ} 07^{\prime} 01^{\prime \prime} \mathrm{O}$ & MG20 & HQ678157 \\
\hline 494 & $\times$ C. leylandii & Novato & California & $38^{\circ} 07^{\prime} 11^{\prime \prime}$ & $122^{\circ} 35^{\prime} 08^{\prime \prime} \mathrm{O}$ & MG13 & HQ678166 \\
\hline 495 & $\times$ C. leylandii & S. Marine & California & $37^{\circ} 52^{\prime} 33^{\prime \prime}$ & $122^{\circ} 26^{\prime} 42^{\prime \prime} \mathrm{O}$ & MG13 & HQ678167 \\
\hline 497 & $\times$ C. leylandii & Knoll Forest & California & $38^{\circ} 00^{\prime} 56^{\prime \prime}$ & $122^{\circ} 41^{\prime} 06^{\prime \prime} \mathrm{O}$ & MG15 & HQ678168 \\
\hline 499 & Cupressus sempervirens & Tiburon & California & $37^{\circ} 52^{\prime} 59^{\prime \prime}$ & $122^{\circ} 28^{\prime} 14^{\prime \prime} \mathrm{O}$ & MG16 & HQ678170 \\
\hline 492 & $\times$ Cupressocyparis leylandii & Ukiah & California & $39^{\circ} 05^{\prime} 25^{\prime \prime}$ & $123^{\circ} 13^{\prime} 15^{\prime \prime} \mathrm{O}$ & MG12 & HQ678165 \\
\hline 498 & $\times$ C. leylandii & Calistoga & California & $38^{\circ} 34^{\prime} 24^{\prime \prime}$ & $122^{\circ} 34^{\prime} 25^{\prime \prime} \mathrm{O}$ & MG1 & HQ678169 \\
\hline 486 & $\times$ C. leylandii & San Diego & California & $32^{\circ} 45^{\prime} 54^{\prime \prime}$ & $117^{\circ} 13^{\prime} 42^{\prime \prime} \mathrm{O}$ & MG20 & HQ678159 \\
\hline 487 & Cupressus macrocarpa & San Diego & California & $32^{\circ} 45^{\prime} 54^{\prime \prime}$ & $117^{\circ} 13^{\prime} 42^{\prime \prime} \mathrm{O}$ & MG21 & HQ678160 \\
\hline 478 & $\times$ Cupressocyparis leylandii & Lodi & California & $38^{\circ} 03^{\prime} 26^{\prime \prime}$ & $121^{\circ} 15^{\prime} 01^{\prime \prime} \mathrm{O}$ & MG9 & $\ldots$ \\
\hline 479 & $\times$ C. leylandii & Lodi & California & $38^{\circ} 03^{\prime} 26^{\prime \prime}$ & $121^{\circ} 15^{\prime} 01^{\prime \prime} \mathrm{O}$ & MG3 & HQ678151 \\
\hline 480 & J. chinensis & Lodi & California & $38^{\circ} 03^{\prime} 27^{\prime \prime}$ & $121^{\circ} 13^{\prime} 24^{\prime \prime} \mathrm{O}$ & MG8 & HQ678153 \\
\hline 481 & $\times$ C. leylandii & Lodi & California & $38^{\circ} 03^{\prime} 29^{\prime \prime}$ & $121^{\circ} 16^{\prime} 39^{\prime \prime} \mathrm{O}$ & MG14 & HQ678154 \\
\hline 482 & $\times$ C. leylandii & Lathrop & California & $37^{\circ} 49^{\prime} 33^{\prime \prime}$ & $121^{\circ} 17^{\prime} 11^{\prime \prime} \mathrm{O}$ & MG18 & HQ678155 \\
\hline 488 & Cupressus macrocarpa & Millbrae & California & $37^{\circ} 32^{\prime} 50^{\prime \prime}$ & $122^{\circ} 22^{\prime} 24^{\prime \prime} \mathrm{O}$ & MG19 & HQ678161 \\
\hline 489 & $\times$ Cupressocyparis leylandii & Millbrae & California & $37^{\circ} 33^{\prime} 55^{\prime \prime}$ & $122^{\circ} 23^{\prime} 15^{\prime \prime} \mathrm{O}$ & MG20 & HQ678162 \\
\hline 490 & $\times C$. leylandii & Hillsborough & California & $37^{\circ} 33^{\prime} 25^{\prime \prime}$ & $122^{\circ} 22^{\prime} 47^{\prime \prime} \mathrm{O}$ & MG6 & HQ678163 \\
\hline 491 & Cupressus macrocarpa & Millbrae & California & $37^{\circ} 33^{\prime} 32^{\prime \prime}$ & $122^{\circ} 22^{\prime} 57^{\prime \prime} \mathrm{O}$ & MG2 & HQ678164 \\
\hline
\end{tabular}


both: 0 to 0.1 (mean $0.01, \Delta 0.01$, bins 200) for $\theta$ and 0 to 1,000 (mean 100, $\Delta 100$, bins 200) for $M$. The number of recorded steps after burn-in was 5,000.

\section{RESULTS}

Based on morphology (data not shown), the isolates employed in all aspects of this study were considered to be $S$. cardinale. Analysis of a portion of the $\beta$-tubulin locus for a randomly selected subset of these isolates confirmed that putative $S$. cardinale isolates in this study, independent of provenance, belonged to the same species, and they all closely or perfectly matched GenBank accessions of $S$ cardinale isolates. All $S$. cardinale isolates were clearly distinct from $S$. unicorne and $S$. cupressi, and isolates putatively assigned to one of the three species fell into three statistically supported clades in both the NJ and ML analyses. NJ results are shown in Figure 1 (TreeBASE Study 11435). Both NJ and ML analyses revealed the presence of two distinct $\beta$-tubulin haplotypes within $S$. cardinale, and both were present in California, whereas a single haplotype was present among isolates from the Mediterranean. Sequences of two iso- lates, one from Chile and one from New Zealand, perfectly matched the sequence of the second California haplotype, which is absent from the Mediterranean (Fig. 1) (GenBank accession numbers HQ678145 to HQ678171, JF271675 to JF27167).

The $I_{A} S$ calculated for the California and Mediterranean populations (excluding Morocco) using SSR data were strikingly different. Whereas association among alleles appeared to be random in the California population $\left(\mathrm{I}_{\mathrm{A}}=-0.041, P=0.556\right)$, a significant $\left(\mathrm{I}_{\mathrm{A}}=0.585, P=0.009\right) \mathrm{LD}$ was found for the Mediterranean population. The Stoddart and Taylor's Index $(G)$, clonal genotype diversity $(R)$, and expected heterozygosity $\left(H_{\mathrm{E}}\right)$ indices are shown in Table 2 and were always much higher for the Californian than for the Mediterranean population.

In total, 46 MGs were identified: 20 in California, 25 in the Mediterranean region, and 1 in Morocco. Within the Mediterranean region, $14 \mathrm{MGs}$ were from Italy, 10 were exclusively from outside Italy (Algeria, France, Greece, and Portugal), and 6 were found both in and outside Italy (Algeria, France, Greece, and Spain). In all, 50 isolates belonging to seven MGs were found in locations at great distances from one another both in California ( 2 isolates from one MG in the San Francisco Bay Area and San

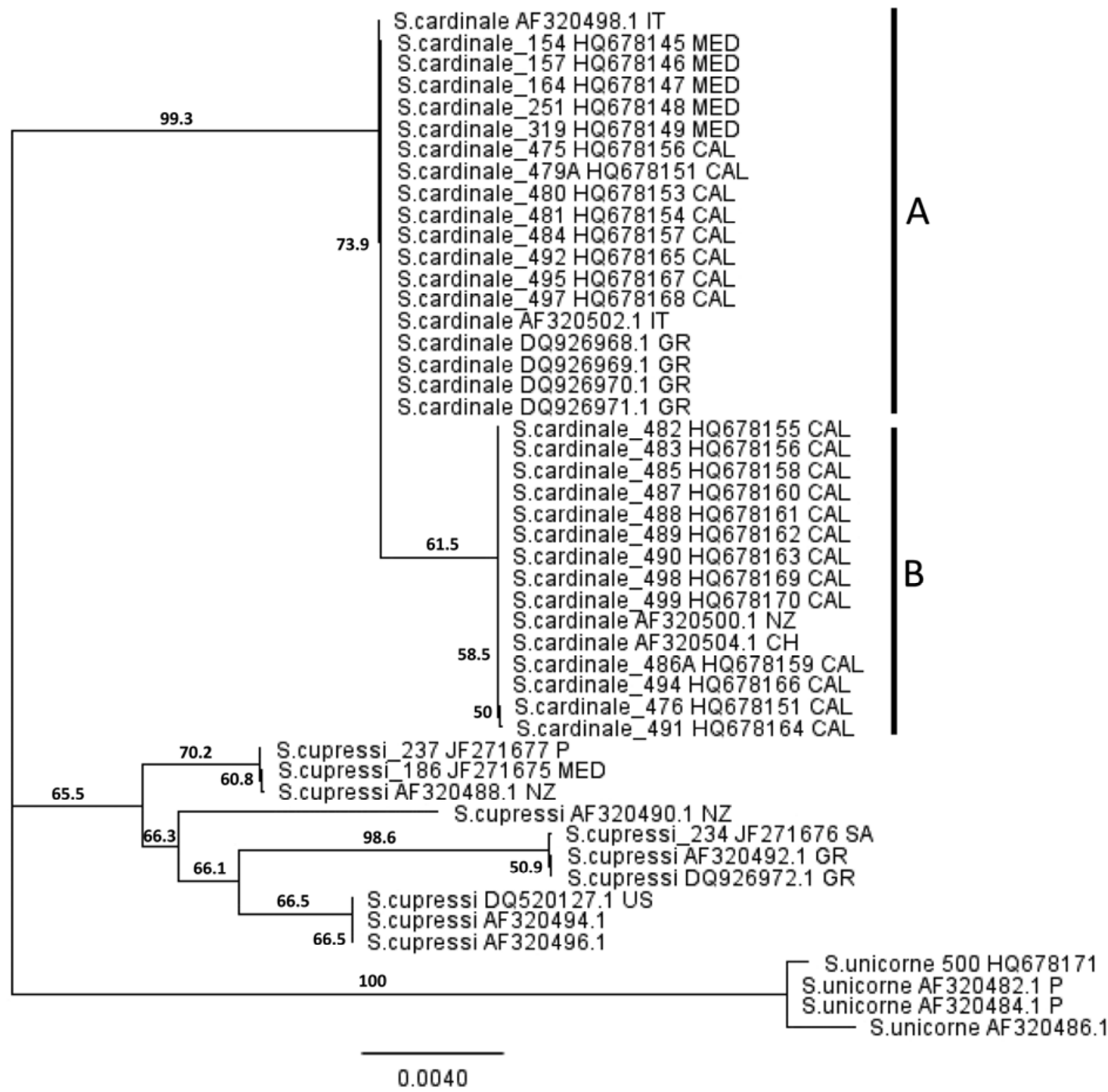

Fig. 1. Neighbor-joining tree based on Seiridium cardinale $\beta$-tubulin sequences from Californian and Mediterranean samples together with sequences from Chile and New Zealand from GenBank, and using S. unicorne and S. cupressi as outgroups. CH = Chile, IT = Italy, GR = Greece, MED = Mediterranean, NZ = New Zealand, $\mathrm{P}=$ Portugal, $\mathrm{SA}=$ South Africa, $\mathrm{CAL}=$ California, $\mathrm{US}=$ United States. Two different haplotypes $(\mathrm{A}$ and $\mathrm{B})$ within $S$. cardinale are indicated. 
Diego County) and in the Mediterranean at distances $>1,000 \mathrm{~km}$ and in regions separated by the Mediterranean Sea (48 isolates representing six MGs found in Spain and Greece or in Algeria, Greece, and Italy). However, no overlap between genotypes was found between California, Morocco, and the remaining countries sampled in the Mediterranean region. The MSN (Fig. 2) shows the presence of three distinct clusters of MGs in California, Morocco, and the Mediterranean region and indicates that the Mediterranean and Moroccan genotypes are each independently linked to Northern California genotypes. The vast Mediterranean infestation appears to have been generated from a single sequence of individual genotypes, all exclusively from central Italy: genetic Bruvo distance (bd) between the MG ancestral to the entire Mediterranean population (MG26) and its closest California relative (MG10) is small ( $0.286 \mathrm{bd})$ and within the range of distances among MGs within both California ( 0.071 to $0.286 \mathrm{bd}$ ) and the Mediterranean (with the exclusion of the Moroccan infestation) ( 0.071 to $0.348 \mathrm{bd})$. By contrast, the distance of the Moroccan genotype to its closest California relative is somewhat larger (0.393 bd), possibly indicating lack of sampling of intermediate genotypes.

Coalescent analysis detected only minimal migration between California and Greece or Italy, independent of direction, whereas a very significant migration was calculated from Italy into

TABLE 2. Indices of genetic diversity ${ }^{\mathrm{a}}$

\begin{tabular}{lcrc}
\hline Population & $R$ & \multicolumn{1}{c}{$G$} & $H_{\mathrm{E}}$ \\
\hline All & 0.474 & 8.991 & 0.413 \\
California & 0.864 & 12.903 & 0.425 \\
Mediterranean (without Morocco) & 0.348 & 0.629 & 0.239 \\
\hline
\end{tabular}

a $R=$ clonal genotype diversity, $G=$ Stoddart and Taylor's index, and $H_{\mathrm{E}}=$ gene diversity (expected heterozygosity).
Greece. A lower but significant value of migration back into Italy from Greece was also obtained (Table 3).

\section{DISCUSSION}

Results of this study confirm the existence of three distinct Seiridium spp.: $S$. cardinale, $S$ unicorne, and $S$ cupressi. Additionally, the study identified previously unknown genetic variability within $S$. cardinale and suggests that California may be the source of the epidemic in the Mediterranean.

Two $\beta$-tubulin alleles were detected for the first time within this locus and both were present in California. Only one of these alleles was found in the well-represented Mediterranean population, while the other allele was found in California and in two samples from Chile and New Zealand. Unfortunately, the minimal sampling intensity does not allow us to exclude the presence of both clades in countries of the Southern Hemisphere; however, this finding is the first to highlight significant molecular difference, even if at a single locus, within this species.

In general, higher genetic diversity, is expected in source populations of a pathogen $(23,35,37,41,42)$ compared with introduced populations, which have obviously undergone the genetic bottleneck of the founding effect. However, genotypic diversity may become higher than expected in the case of unchecked expansion of populations of introduced pathogens colonizing naïve ecosystems $(11,38)$. We hypothesized that the epidemic levels observed in the Mediterranean may have led to a large number of closely related genotypes but that indices of diversity should remain higher in California, were that state to be a possible source of the pathogen. The SSRs employed in this study were polymorphic and detected a total of 46 MGs: 25 in the Mediterranean (excluding the Moroccan single genotype) and 20 in California. However, the Mediterranean region was sampled much more

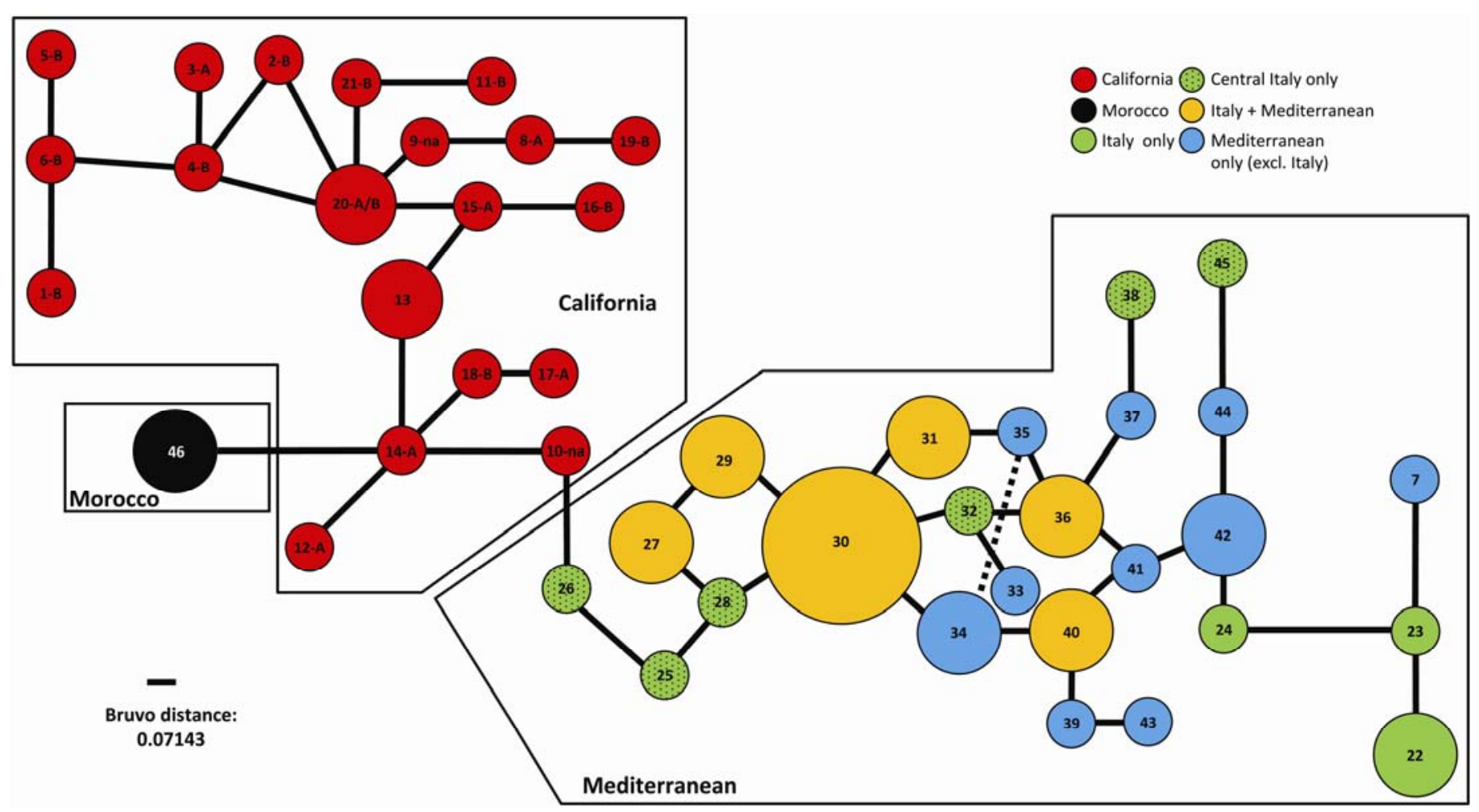

Fig. 2. Minimum spanning network (MSN) showing relationships between multilocus genotypes (MGs) in Californian and Mediterranean populations. Each node represents a different MG and nodes are scaled to reflect the numbers of individuals sharing the same MGs: small $=$ singleton $M G$, medium $=2$ to 5 individuals with same MG, large $=30$ individuals with same MG. Labeling with A, B, or na within nodes relates to whether individuals with that MG were found within clade A or B or not included in the analysis, respectively, in the Seiridium cardinale neighbor-joining tree (Fig. 1). Dotted line between nodes 34 and 35 indicates where the branch length was unable to be scaled properly without overlap. Distance is the equivalent of the shortest distances in the network; for example, equal length to those between 30 and 32 or 32 and 36 . 
extensively than California. Because indices of genotypic and genetic diversity take into consideration sampling intensity, all three indices calculated in this study were significantly higher for California than for the Mediterranean region, indicating California as a likely source for the pathogen.

In most countries affected by the canker pandemic, no sexual stage of the fungus has ever been described, and the pathogen is regarded as reproducing exclusively asexually. However, the sexual stage of this pathogen was incompletely described, once, in California (33). We used SSR data to determine whether LD (i.e., the absence of random assortment among unlinked alleles) may result due to the lack of sexual reproduction. Calculations of the $I_{A}$, surprisingly, revealed that LD was only present in the Mediterranean population and that the allelic distribution of the California population did not show signs of LD, suggesting that sexual reproduction may be ongoing in this U.S. state. The fact that the same genotype was only found twice at significant distances in California as opposed to 48 times in the Mediterranean is also indicative of a different reproductive strategy in the two regions of the world. An alternative, albeit less conservative, explanation of the Californian $\mathrm{I}_{\mathrm{A}}$ may be that multiple introductions of significantly different genotypes and both mating types may have occurred from a third location into California.

Because relationships among individuals within species (or at least within metapopulations belonging to a species) cannot properly be visualized by distance-based approaches resulting in bifurcating representations, we opted to analyze our SSR data through a network analysis. All isolates from California and all isolates from the Mediterranean (excluding the Moroccan genotype) formed two distinct clusters of genotypes. The Moroccan genotype represented a third, distinct "Moroccan" cluster. Both the Mediterranean and the Moroccan genotypes were independently linked to the California cluster. Although no overlap of identical genotypes was found among the three clusters, distance between Californian and genotypes from the other two regions was comparable with distances detected between genotypes within region. A single connection between the Californian and Mediterranean clusters through a series of three genotypes exclusively present in central Italy is best interpreted as depicting a single and not a multiple introduction of $S$. cardinale from California into central Italy. An alternative explanation of the data could be provided by convergence of genotypes from two distinct introductions from a third region leading through random genetic drift processes to two extremely closely related genotypes, one in California and one in central Italy. In order for this hypothetical convergent microevolutionary process to have occurred, convergent evolution would have to have occurred independently at all loci employed in this analysis. We decided to compare the probability $(P)$ of these two alternative hypotheses; namely, the direct emergence of the basal Mediterranean genotype from a California genotype $\left(\mathrm{H}_{1}\right)$ and the convergent evolution of two genotypes from two independent founder genotypes $\left(\mathrm{H}_{2}\right)$. We opted to actually favor $\mathrm{H}_{2}$ by not considering independent evolution of each SSR locus but assuming that the only ecologically possible genotypes were the ones depicted in our network (Fig. 2). We assumed the founder MG of each infestation would be (i) very common, (ii) the progenitor of a large number of other geno- types, and (iii) situated somewhere in the center of the network for each region (i.e., MG20 for California and MG30 for the Mediterranean). We then calculated the probability $(P)$ that from those two founder genotypes, MG10 and MG26 (i.e., the two closely related genotypes in the two regions of the world), would arise independently for California and the Mediterranean, respectively, by multiplying the $P$ of generating the next MG in the chain of genotypes between MG20 and MG10 for California and between MG30 and MG26 for the Mediterranean. At each step, the $P$ of generating the "correct" next MG was calculated as $P_{\text {next }}=1 / n$ MG derived from progenitor.

For each region, the overall $P_{\text {reg }}$ was calculated by multiplying the $P$ of one MG by the $P$ of the next, and so on, until MG10 and MG26 were generated, starting from the founder genotypes for each region, as follows: $P_{\text {cal }}=0.2 \times 0.33 \times 0.5 \times 0.2 \times 0.5=$ 0.0033 and $P_{\text {med }}=0.2 \times 0.33 \times 0.5=0.033$.

The probability $(P)$ of convergent evolution of two closely related genotypes from two independent sources is $P_{\mathrm{H} 2}=P_{\text {cal }} \times$ $P_{\text {med }}=0.0033 \times 0.33=0.0001$.

Alternatively, if we accept that MG26 in Italy is a direct descendant of MG10 in California, then $P_{\mathrm{H} 1}=0.5$.

Of course, if convergent evolution at each of the seven loci were to be considered, the difference between probabilities associated with $\mathrm{H}_{1}$ and $\mathrm{H}_{2}$ would increase by several orders of magnitude. We conclude that, at this point, an introduction from California into Italy is the best explanation of the data. Additionally, preliminary analysis of $>200$ amplified fragment length polymorphism markers (G. Della Rocca, unpublished data) shows an extremely close relatedness of isolates from California and central Italy, with Californian isolates being basal to Italian isolates, further supporting our interpretation of the SSR data here presented.

In the case of the sudden oak death pathogen, Phytophthora ramorum, putative source genotypes were the most abundant and were additionally identified as direct progenitors of multiple new MGs, generated through mitotic recombinations or mutations $(38,39)$. In the case of $S$. cardinale, it appears that epidemic expansion of the pathogen started with the emergence of MG28 and not with the original founder genotype, MG26 (Fig. 2). This difference may be due to the introduction of a genotype already preadapted to the ecological conditions of the novel environment and to favorable weather conditions in the case of $P$. ramorum, whereas a longer lag phase may have occurred for $S$. cardinale due to either the need for adaptation or the presence of unfavorable weather conditions immediately after the introduction of the pathogen in the Mediterranean region.

Network analysis suggests that introduction of the pathogen into the Mediterranean did not occur multiple times whereas movement within the Mediterranean may have occurred repeatedly, given the frequent retrieval of the same genotype in distant locations. We performed coalescent analysis to estimate migration among regions and potentially validate the results suggested by the network analysis. Only the three well-sampled populations from California, Italy, and Greece were included in this analysis, to avoid biases caused by small population size. Results of MIGRATE-N indicated a lack of significant migration between California and Italy or Greece, as expected of an epidemic origi-

TABLE 3. Results of coalescent analysis for California, Greece, and Italy populations using Migrate-N

\begin{tabular}{|c|c|c|c|c|c|c|c|c|c|c|c|}
\hline \multirow[b]{2}{*}{ Location } & \multicolumn{5}{|c|}{$\theta$} & \multicolumn{6}{|c|}{ Migration rate } \\
\hline & $2.50 \%$ & Mode & $97.50 \%$ & Median & Mean & Direction & $2.50 \%$ & Mode & $97.50 \%$ & Median & Mean \\
\hline \multirow{2}{*}{ 1, Greece } & 0.073 & 0.098 & 0.100 & 0.091 & 0.088 & $1 \rightarrow 2$ & 0 & 22.5 & 45 & 27.5 & 23.16 \\
\hline & $\ldots$ & $\ldots$ & $\ldots$ & $\ldots$ & $\ldots$ & $1 \rightarrow 3$ & 0 & 2.5 & 20 & 12.5 & 5.142 \\
\hline \multirow[t]{2}{*}{2 , Italy } & 0.094 & 0.098 & 0.100 & 0.098 & 0.097 & $2 \rightarrow 1$ & 0 & 42.5 & 135 & 462.5 & 347.45 \\
\hline & $\ldots$ & $\ldots$ & $\ldots$ & $\ldots$ & $\ldots$ & $2 \rightarrow 3$ & 0 & 2.5 & 25 & 12.5 & 6.18 \\
\hline \multirow[t]{2}{*}{ 3, California } & 0.094 & 0.098 & 0.100 & 0.098 & 0.097 & $3 \rightarrow 1$ & 0 & 2.5 & 25 & 12.5 & 6.392 \\
\hline & $\ldots$ & $\ldots$ & $\ldots$ & $\ldots$ & $\ldots$ & $3 \rightarrow 2$ & 0 & 2.5 & 25 & 12.5 & 6.886 \\
\hline
\end{tabular}


nated by a single introduction event. However, high migration was detected from Italy into Greece and lower but significant migration was also detected from Greece back into Italy. These high migration values are likely to be justified by repeated movement of infected plant material within the Mediterranean. An asymmetry in migration levels is expected during the initial phases of an invasion characterized by movement mainly from the area of original establishment (e.g., Italy) into areas being subsequently colonized (e.g., Greece).

We believe that data overall indicate that California is the most likely source of the epidemic in the Mediterranean, with two distinct introduction events, one in Italy and one in Morocco. After a lag phase in central Italy, populations of the pathogen expanded in size and started being moved across southern Europe, most likely in association with the movement of plants by humans. The magnitude of migration from Italy into Greece is in agreement with central Italy being at the source of the Mediterranean epidemic: pathogen populations are likely to have been greatly favored by the high density of susceptible $C$. sempervirens in central Italy. In contrast, the low density of hosts in Morocco (and perhaps a later date of introduction) may have slowed the epidemic linked to that introduction. Incidentally, our genetic reconstruction based on a variety of approaches depicts a scenario that well matches the history of the disease, which was first reported in California, then in central Italy, and then in other parts of Italy and the Mediterranean $(2,15,17,18,25,31,65,67,69,70,75)$.

The presence of higher genetic diversity in California, including two clearly distinct $\beta$-tubulin alleles, and the finding that genotypes from the Southern Hemisphere display at least some genetic differences with European isolates, suggests that care should be taken to avoid further introductions of $S$. cardinale into the Mediterranean basin from either California or the Southern Hemisphere. Likewise, further introductions of $S$. cardinale from California and the Mediterranean into countries of the Southern Hemisphere should be avoided, if broader sampling confirms the homogeneity of pathogen populations in those countries. Although the exact relationship between genotypic and phenotypic variability is currently unknown, ecological fitness, pathogenicity, and potentially mating type (given the lack of LD reported in this study for California) are all likely to be correlated to genotypic variability. Consequently, the introduction of genetically different individuals in areas where the pathogen was previously introduced may result in a shift in the course of the epidemic, with dramatic impacts on host populations. Such shifts have been reported in other pathosystems in both the presence or absence of sexual reproduction $(13,29,52)$. Unfortunately, regulatory systems of most countries often do not easily allow for a distinction between groups of genotypes of the same species, even when genetic information points to a significant isolation and independent evolution of such groups. In the case of cypress canker in the Mediterranean region, the introduction and establishment of significantly different genotypes or of genotypes bearing a different mating allele would also nullify the utility of resistant cypress lines selected to tolerate the closely related genotypes currently established in the Mediterranean. The discovery of California as a center of diversity for $S$. cardinale allows for the design of further selection trials using genotypes that are clearly distinct from the ones currently present in the Mediterranean. However, in order to ensure the durability of resistant host lines, it is imperative to minimize both the introduction of different genotypes and the potential for sexual recombination. Prevention of the introduction of novel mating alleles requires regulations to minimize or halt any further introductions of genetically distinct genotypes.

Given the good spatial coverage of the sampling conducted in the Mediterranean basin, the presence of identical genotypes in locations separated by thousands of kilometers and by large bodies of water is unlikely to be justified by natural spread of the pathogen and is most likely associated with movement of infected plant material by humans. Airborne spread of multicelled conidia at large distances is unreported for this type of fungal pathogen and, although movement of infected plant parts on migratory birds cannot be excluded, it is extremely less likely than human induced movement, given that Cypress trees are not known as significant foraging or nesting species for any migratory species. Although spread through human trade is not unexpected, the large number of findings $(n=50)$ of identical but geographically distant genotypes indicates that humans are moving the pathogen quite frequently. It is important to fully understand how and through which routes human activities are facilitating the long-range dispersal of this serious pathogen to prevent further introductions. The fact that the establishment of a single genotype may have caused the entire Mediterranean infestation highlights the absolute need and the challenge of preventing any further introduction. Although the genetic relatedness of Californian and Mediterranean populations is well supported by our data, sampling from other regions of the world was only minimal. Hence, although California appears to be the most likely source of the epidemic in the Mediterranean, whether it is the worldwide source of $S$. cardinale remains a distinct possibility but needs to be verified by further comparison with populations from other regions of the world. In the meanwhile, a closer look at the genotypic and phenotypic variability of Californian populations may help better understand the Mediterranean epidemic. Finally, additional research needs to confirm whether and where sexual reproduction may be occurring in California. Certainly, the absence of epidemics in the natural range of some Californian Cupressus spp., including C. macrocarpa, C. bakeri, C. macnabiana, C. forbesii, and $C$. pygmaea, is suggestive of a long co-evolutionary process between hosts and pathogen.

\section{ACKNOWLEDGMENTS}

This study would have not been possible without assistance of countybased personnel from the University of California Cooperative Extension and the California State. We thank B. Black-Rogers, S. Swain, L. Costello, D. Shaw, and P. Nolan; the Quail Botanical Garden in San Diego for allowing us to sample trees in their grounds; V. Di Leonardo, was instrumental for the collection of isolates in the Mediterranean region; and T. Osmundson for helping with the phylogenetic analyses.

\section{LITERATURE CITED}

1. Agapow, P. M., and Burt, A. 2001. Indices of multilocus linkage disequilibrium. Mol. Ecol. Notes 1:101-102.

2. Anastassiades, B. 1963. A new for Greece disease of the Cypress. Ann. Inst. Phytopathol. Benaki (N.S.) 5:164-166.

3. Andreoli, C., Panconesi, A., Ponchet, J., and Raddi, P. 1984. Etude comparée du comportement des cyprès a Florence et a Antibes. Pages 920 in: Maladie du cyprès (Coryneum cardinale). Rep. EUR 9200 EN-FRIT. J. Ponchet, ed. Luxembourg.

4. Arguedas Gamboa, M. 1996. Inventory of diseases in forest species in Costa Rica. Rev. For. Centroam. 5:20-24.

5. Barnes, I., Roux, J., and Wingfield, M. J. 2001. Characterization of Seiridium spp. associated with Cypress Canker based on $\beta$-tubulin and histone sequences. Plant Dis. 85:317-321.

6. Barthelet, J., and Vinot, M. 1944. Notes sur les maladies des cultures méridionales. Ann. Epiphyties 10:18-20.

7. Beerli, P. 2006. Comparison of Bayesian and maximum likelihood inference of population genetic parameters. Bioinformatics 22:341-345.

8. Beerli, P. 2009. How to use migrate or why are Markov chain Monte Carlo programs difficult to use? Pages 42-79 in: Population Genetics for Animal Conservation, Conservation Biology 17. G. Bertorelle, M. W. Bruford, H. C. Hauffe, A. Rizzoli, and C. Vernesi, eds. Cambridge University Press, Cambridge.

9. Beerli, P., and Felsenstein, J. 2001. Maximum likelihood estimation of a migration matrix and effective population sizes in subpopulations by using a coalescent approach. Proc. Natl. Acad. Sci. USA 98:4563-4568.

10. Benson, D. A., Karsch-Mizrachi, I., Lipman, D. J., Ostell, J., and Wheeler, D. L. 2008. GenBank. Nucleic Acids Res. 36:25-30.

11. Bergemann, S., Smith, A., Parrent, J., Gilbert, G., and Garbelotto, M. 2009. Genetic population structure and distribution of a fungal polypore, 
Datronia caperata in mangrove forests of Central America. J. Biogeogr. 36:266-279.

12. Birch, T. T. C. 1933. Gummosis diseases of Cupressus macrocarpa. N.Z. J. For. 3:108-113.

13. Brasier, C. M., Cooke, D. E. L., and Duncan, J. M. 1999. Origins of a new Phytophthora pathogen through interspecific hybridization. Proc. Natl. Acad. Sci. USA 96:5878-5883.

14. Bruvo, R., Michiels, N. K., D’Souza, T. G., and Schulenburg, H. 2004. A simple method for the calculation of microsatellite genotype distance irrespective of ploidy level. Mol. Ecol. 13:2101-2106.

15. Caetano, M. F. F. 1980. Uma grave doença das Cupressáceas em Portugal. Agros 63:5-10.

16. Chou, C. K. S. 1989. Morphological and cultural variation of Seiridium spp. from cankered Cupressaceae hosts in New Zealand. Eur. J. For. Pathol. 18:435-445.

17. Cvjetković, B., and Glavaš, M. 1978. Coryneum cardinale Wag. causing of the necrosis of the bark and dieback of the branches of the cypress in Yugoslavia. Zast. Bilja 29:365-370.

18. Danti, R., Della Rocca, G., and El Wahidi, F. 2009. Seiridium cardinale newly reported on Cupressus sempervirens in Morocco. Plant Pathol. 58:1174.

19. Danti, R., Panconesi, A., Di Lonardo, V., Della Rocca, G., and Raddi, P. 2006. 'Italico' and 'Mediterraneo': Two Seiridium cardinale cankerresistant cypress cultivars of Cupressus sempervirens. HortScience 41:1357-1359.

20. Della Rocca, G., Buonamici, A., Cossu, C., Vendramin, G. G., and Danti, R. 2009. Development and characterization of eight microsatellite markers for the fungus Seiridium cardinale. Mol. Ecol. Resour. 9:1375-1429.

21. Drummond, A. J., Ashton, B., Buxton, S., Cheung, M., Cooper, A., Duran, C., Field, M., Heled, J., Kearse, M., Markowitz, S., Moir, R., StonesHavas, S., Sturrock, S., Thierer, T., and Wilson, A. 2011. Geneious v5.4. http://www.geneious.com/

22. Dutech, C., Fabreguettes, O., Capdevielle, X., and Robin, C. 2010. Multiple introductions of divergent genetic lineages in an invasive fungal pathogen, Cryphonecria parasitica, in France. Heredity 105:220-228.

23. Engelbrecht, C. J. B., Harrington, C., Steimel, J., and Capretti, P. 2004. Genetic variation in eastern North American and putatively introduced populations of Ceratocystis fimbriata f. platani. Mol. Ecol. 13:2995-3005.

24. Excoffier, L., and Smouse, P. E. 1994. Using allele frequencies and geographic subdivision to reconstruct gene trees within a species-molecular variance parsimony. Genetics 136:343-359.

25. Faddoul, J. 1973. Contribution a l'etude du Coryneum cardinale Wag. morphologie, biologie, physiologie. These n. 390, Université Paul Sabatier de Toulose.

26. Funk, A. 1974. Microfungi associated with dieback of native Cupressaceae in British Columbia. Can. Plant Dis. Surv. 54:166-168.

27. Gansner, E. R., and North, S. C. 2000. An open graph visualization system and its applications to software engineering. Software Practice Experience 30:1203-1233.

28. Gonthier, P., Nicolotti, G., Linzer, R., Guglielmo, F., and Garbelotto, M. 2007. Invasion of European pine stands by a North American forest pathogen and its hybridization with native interfertile taxon. Mol. Ecol. 7:1389-1400

29. Goodwin, S. B., Sujkowski, L. S., Dyer, A. T., Fry, B. A., and Fry, W. E. 1995. Direct detection of gene flow and probable sexual reproduction of Phytophthora infestans in Northern North America. Phytopathology 85:473-479.

30. Graniti, A. 1998. Cypress canker: A pandemic in progress. Annu. Rev. Phytopathol. 36:91-114.

31. Grasso, V. 1951. Un nuovo agente patogeno del Cupressus macrocarpa Hartw. in Italia. Ital. For. Mont. 6:63-65.

32. Hamelin, R. C., Hunt, R. S., Geils, B. W., Jensen, G. D., Jacobi, V., and Lecours, N. 2000. Barrier to gene flow between eastern and western populations of Cronartium ribicola in North America. Phytopathology 90:1073-1078.

33. Hansen, H. 1956. The perfect stage of Coryneum cardinale. Phytopathology 46:636-637.

34. Hennon, P. E. 1990. Fungi on Chamaecyparis nootkatensis. Mycologia 82:59-66.

35. Ivors, K., Hayden, K. J., Bonants, P. J. M., Rizzo, D. M., and Garbelotto, M. 2004. AFLP and phylogenetic analyses of North American and European populations of Phytophthora ramorum. Mycol. Res. 108:378-392.

36. Krokene, P. 2004. A PCR-RFLP based diagnostic technique to rapidly identify Seiridium species causing cypress canker. Mycologia 96:13521354.

37. Linzer, R. E., Rizzo, D. M., Cacciola, S. O., and Garbelotto, M. 2009. AFLPs detect low genetic diversity for Phytophthora nemorosa and P. pseudosyringae in the US and Europe. Mol. Ecol. 113:298-307.

38. Mascheretti, S., Croucher, P., Kozanitas, M., Baker, L., and Garbelotto, M. 2009. Genetic epidemiology of the sudden oak death pathogen
Phytophthora ramorum in California. Mol. Ecol. 18:4577-4590.

39. Mascheretti, S., Croucher, P. J. P., Vettraino, A., Prospero, S., and Garbelotto, M. 2008. Reconstruction of the sudden oak death through microsatellite analysis of the pathogen Phytophthora ramorum. Mol. Ecol. 11:2755-2768.

40. Meirmans, P. G., and Van Tienderen, P. H. 2004. GENOTYPE and GENODIVE: Two programs for the analysis of genetic diversity of asexual organisms. Mol. Ecol. Notes 4:792-794.

41. Milgroom, M. G., Sotirovski, K., Spica, D., Davis, J. E., Brewer, M. T., Milev, M., and Cortesi, P. 2008. Clonal population structure of the chestnut blight fungus in expanding ranges in southeastern Europe. Mol. Ecol. 17:4446-4458.

42. Milgroom, M. G., Wang, K., Zhou, Y., Lipari, S. E., and Kaneko, S. 1996. Inter-continental population structure of the chestnut blight fungus, Cryphonectria parasitica. Mycologia 88:179-190.

43. Miller, M. A., Pfeiffer, W., and Schwarts, T. 2010. Creating the CIPRES Science Gateway for inference of large phylogenetic trees. Pages 1-8 in: Proc. Gateway Comput. Environ. Workshop (GCE), New Orleans.

44. Mordue, J. E. M. 1976. CMI Descriptions of Pathogenic Fungi and Bacteria, no. 514. CMI, Kew, Surrey, UK

45. Moricca, S., Børja, I., Vendramin, G. G., and Raddi, P. 2000. Differentiation of Seiridium species associated with virulent canker on cypress in the Mediterranean region by PCR-SSCP. Plant Pathol. 49:774781.

46. Mujica, F., and Vergara, C., eds. 1980. Flora fungosa chilena, 2nd ed. E. Oehrens, rev. Publ. Cient. Agric. 5, Universidad de Chile, Santiago, Chile.

47. Mutto, S., and Panconesi, A. 1987. Ultrastructural modifications in Cupressus sempervirens tissues invaded by Seiridium cardinale. Eur. J. For. Pathol.17:193-204.

48. Ocasio-Morales, R. G., Tsopelas, P., and Harrington, T. C. 2007. Origin of Ceratocystis platani on native Platanus orientalis in Greece and its impact on natural forests. Plant Dis. 91:901-904.

49. Panconesi, A. 1990. Pathological disorders in the Mediterranean basin. Pages 54-81 in: Agrimed Res. Programme. Prog. EEC Res. Cypress Dis. Rep. EUR 12493 EN. J. Ponchet, ed. Luxembourg.

50. Panconesi, A., and Raddi, P. 1991. Cancro del cipresso. Aspetti biologici ed epidemiologici. Pages 49-60 in. Il cipresso. CNR, Regione Toscana, Italy.

51. Panconesi, A., Santini, A., Casini, N., and Degl'Innocenti, C. 1995. Seiridium cardinale spread in the woody tissue of Cupressus sempervirens. Shoot and foliage diseases in forest trees. Pages 138-141 in: Proc. IUFRO Joint Meet. Work Parties. Vallombrosa, Firenze, Italy. P. Capretti, U. Heiniger, and R. Stephan, eds. Università di Firenze, Consiglio Nazionale delle Ricerche, Italy.

52. Paoletti, M., Buck, K. W., and Brasier, C. M. 2006. Selective acquisition of novel mating type and vegetative incompatibility genes via interspecies gene transfer in the globally invading eukaryote Ophiostoma novo-ulmi. Mol. Ecol. 15:249-262.

53. Pedron, L., Piva, G., and La Porta, N. 2007. The genetic structure of cypress canker fungus in Italy using RAPD and minisatellite markers. Acta Silvatica Lignaria Hung. Spec. Ed. 159-168.

54. Ponchet, J., and Andreoli, C. 1990. Compartmentalization and reaction in the host. Pages 96-111 in: Agrimed Res. Programme. Prog. EEC Res. Cypress Dis. Rep. EUR 12493 EN. J. Ponchet, ed. Luxembourg.

55. Ponchet, J., Andreoli, C., Xenopoulos, S., Caetano, M. F., Raddi, P., and Panconesi, A. 1990. Pathogenic variability in Seiridium sp. Pages 112-126 in: Agrimed Res. Programme. Prog. EEC Res. Cypress Dis. Rep. EUR 12493 EN. J. Ponchet, ed. Luxembourg.

56. Raddi, P. 1979. Variabilità della resistenza al cancro nell'ambito del cipresso comune (C. sempervirens) e di altre specie. Pages 185-193 in: Il Cipresso: Malattie e difesa. V. Grasso and P. Raddi, eds. CEE, AGRIMED, Firenze, Italy.

57. Raddi, P., and Panconesi, A. 1984. Pathogenicity of some isolates of Seiridium (Coryneum) cardinale, agent of cypress canker disease. Eur. J. For. Pathol.14:348-354.

58. Raddi, P., and Panconesi, A. 1995. Present and future of cypress canker disease research. Shoot and foliage diseases in forest trees. Pages 112-117 in: Proc. IUFRO Joint Meet. Work Parties. Vallombrosa, Firenze, Italy. P. Capretti, U. Heiniger, and R. Stephan, eds. Università di Firenze, Consiglio Nazionale delle Ricerche, Italy.

59. Raddi, P., Panconesi, A., Xenopoulos, S., Ferrandes, P., and Andreoli, C. 1990. Genetic improvement for resistance to canker disease. Pages 127134 in: Agrimed Res. Programme. Prog. EEC Res. Cypress Dis. Rep. EUR 12493 EN. J. Ponchet, ed. Luxembourg.

60. Sanders, I. R. A., Groppe, M., Boller, K., and Wiemken, T. A. 1995. Identification of ribosomal DNA polymorphisms among and within spores of the Glomales: application to studies on the genetic diversity of arbuscular mycorrhizal fungal communities. New Phytol. 130:419-427.

61. Saravì Cisneros, R. 1953. Cancrosis de los Cipreses provocada por Coryneum cardinale Wag. en la provincia de Buenos Aires (Argentina). 
Rev. Fac. Agron. Univ. Nac. La Plata 29:107-119.

62. Sinclair, W. A., and Lyon, H. H., eds. 2005. Diseases of Trees and Shrubs, 2nd ed. Comstock Publishing Associates, Ithaca and London.

63. Solel, Z., Messinger, R., Golan, Y., and Madar, Z. 1983. Coryneum canker of cypress in Israel. Plant Dis. 67:550-551.

64. Stamatakis, A. 2006. RAxML-VI-HPC: Maximum likelihood-based phylogenetic analyses with thousands of taxa and mixed models. Bioinformatics 22:2688.

65. Stoddart, J. A., and Taylor, J. F. 1988. Genotypic diversity: Estimation and prediction in samples. Genetics 118:705-711.

66. Strouts, R. G. 1970. Coryneun canker of Cupressus. Plant Pathol. 19:149150.

67. Sumer, S. 1987. The distribution of cypress (Cupressus sempervirens L.) in Turkey and the current status in its pests and diseases, especially cypress canker disease. Istanbul Univ. Ormanm Fak. Derg. Seri A 37:46-66.

68. Sutton, B. C., and Gibson, I. A. S. 1972. Seiridium cardinale. CMI Description of Pathogenic Fungi and Bacteria N. 326. CMI, Kew, Surrey, UK.

69. Torres, J. 1969. Grave enfermedad de los cipreses en España. Bol. Serv. Plagas For. (Spain) 12:97-99.
70. Tsopelas, P., Angelopoulos, A., and Nikolaou, K. 2008. Seiridium cardinale is a new threat to cypress trees in Cyprus. Plant Pathol. 57:784.

71. Tsopelas, P., Barnes, I., Wingfield, M. J., and Xenopoulos, S. 2007. Seiridium cardinale on Juniperus species in Greece. For. Pathol. 37:338-347.

72. Urbasch, I. 1993. Natural occurrence of Seiridium cardinale on Thuja in Germany. J. Phytopathol. 137:189-194.

73. Viljoen, C. D., Wingfield, B. D., and Wingfield, M. J. 1993. Comparison of Seiridium isolates associated with cypress canker using sequence data. Exp. Mycol. 17:323-328.

74. Wagener, W. W. 1928. Coryneum canker of cypress. Science 67:584.

75. Wagener, W. W. 1939. The canker of Cupressus induced by Coryneum cardinale n. sp. J. Agric. Res. 58:1-46.

76. Wingfield, M. J., and Swart, W. J. 1988. Cypress canker in South Africa. Page 361 in: Abstr. 5th Int. Congr. Plant Pathol. Kyoto, Japan.

77. Xenopoulos, S. 1991. Pathogenic variability of various isolates of Seiridium cardinale, S. cupressi and S. unicorne inoculated on selected Cupressus clones and seedlings. Eur. J. For. Pathol. 21:129-135.

78. Xenopoulos, S., and Diamandis, S. 1985. A distribution map for Seiridium cardinale causing the cypress canker in Greece. Eur. J. For. Pathol. 15:223-226. 